\title{
REVISIÓN \\ Genética de la esquizofrenia: avances en el estudio de genes candidatos
}

Adriana Pacheco \& Henriette Raventós

CIBCM, Universidad de Costa Rica, 2060 San José, Costa Rica.

Recibido 23-VII-2004. Corregido 05-IX-2004. Aceptado 05-IX-2004.

\begin{abstract}
Genetics of Schizophrenia: advances in the study of candidate genes. Schizophrenia is one of the most severe mental disorders that affect $1 \%$ of the population worldwide. It is clear that both genetic and environmental factors participate in its etiology. Nonetheless, the effort to identify susceptibility genes has been difficult and there are no unequivocal findings until now. Notwithstanding this, during the last couple of years, a group of candidate genes has been identified because of their possible role in the physiopathology or by association and linkage studies. In this article, the role of these genes is summarized as well as the results of the studies conducted in Costa Rica by our group. Rev. Biol. Trop. 52(3): 467-473. Epub 2004 Dic 15.
\end{abstract}

Keywords: Schizophrenia, susceptibility genes, candidate genes, Costa Rica.

Palabras clave: Esquizofrenia, genes de susceptibilidad, genes candidatos, Costa Rica.

La esquizofrenia es una de las enfermedades mentales severas con trastornos en el pensamiento (Evans et al. 2001, Adityanjee et al. 1999). Afecta a un 1\% de la población mundial. Se ha sugerido que su prevalencia varía de acuerdo al país, por ejemplo en Taiwán es de $0.3 \%$ y en una población aislada de Finlandia es de un $3 \%$. (Mowry y Nancarrow, 2001). Sin embargo, otros autores consideran que no existen diferencias reales entre poblaciones. Se manifiesta tanto en hombres como en mujeres (Berretini 2000). Es una enfermedad crónica y permanente a lo largo de la vida. Generalmente se inicia al final de la adolescencia o en adultos jóvenes. Ocurre en todos los grupos socioeconómicos. Sin embargo, las manifestaciones clínicas de la enfermedad, las hospitalizaciones y los efectos secundarios de los medicamentos dificultan la incorporación laboral de las personas con esquizofrenia, lo que disminuye sus ingresos (Cowan et al. 2002).

El diagnostico es complejo. El cuadro clínico incluye síntomas positivos (aumento de la actividad cerebral) y negativos (disminución de la actividad cerebral). Se consideran síntomas positivos las alucinaciones (auditivas, visuales u olfatorias), los delirios y la desorganización del pensamiento, y síntomas negativos, el aislamiento social, la disminución de la comunicación y del contacto visual y la ausencia de expresiones faciales. Según el Manual Diagnostico de la Asociación Psiquiátrica de los Estados Unidos (DSM-IV), cierta combinación de síntomas deben estar presentes por al menos 6 meses para hacer el diagnostico (American Psychiatric Association 1994).

\section{ETIOLOGÍA}

La etiología de las enfermedades mentales severas es compleja. Participan tanto factores genéticos como ambientales. Entre los factores ambientales se han relacionado la zona de residencia, complicaciones obstétricas, factores infecciosos, entre otros. Los individuos que 
viven en zonas urbanas tienen un riesgo 35 veces mayor de desarrollar la enfermedad. Las complicaciones obstétricas pre- y peri-natales tales como incompatibilidad $\mathrm{Rh}$, bajo peso al nacer y deficiencias nutricionales de la madre en el primer trimestre, se han asociado con el riesgo posterior de desarrollar la enfermedad (Nelly y Murria 2000). Se ha sugerido que estos factores pueden interferir con el desarrollo de la proliferación celular y la migración neuronal en el sistema nervioso (Torrey et al. 1994, Bahn 2002, Nelly y Murria 2000).

También se han estudiado factores infecciosos como la exposición al virus de la influenza en el segundo trimestre de embarazo. Se ha observado un aumento de nacimientos en invierno en las personas que presentan la enfermedad, lo cual también sugiere una etiología infecciosa (Torrey et al. 1994).

La heredabilidad de la esquizofrenia es de alrededor de un 50\% comparado al desorden bipolar que es de un $65 \%$ aproximadamente (Bahn 2002). Un familiar en primer grado de una persona afectada con esquizofrenia tiene un riesgo diez veces mayor de padecer la enfermedad comparado a la población general. El riesgo para los familiares en primer grado oscila entre un $9 \%$ y un $16 \%$. El hijo de dos personas afectadas tiene un $46 \%$ de riesgo de presentar la enfermedad. (Faraone et al. 2002).

La concordancia entre gemelos monocigóticos es de un $46-53 \%$ y entre los gemelos dicigóticos de un $10-15 \%$. Se ha observado que la probabilidad de que el hijo de un gemelo monocigótico no afectado presente la enfermedad es el mismo al riesgo del hijo del gemelo afectado. Los estudios con adopciones también demuestran que el aumento del riesgo se debe principalmente a factores genéticos. Sin embargo, como los gemelos monocigóticos no son siempre concordantes, los genes no son los únicos que participan en el desarrollo de la enfermedad. (Faraone et al. 2002)

Probablemente la esquizofrenia no es producto de un factor o gen único. El riesgo entre familiares sugiere que la enfermedad se transmite por la herencia de un número de genes de efecto menor y es posible la participación de un gen de efecto mayor de herencia recesiva (Risch 1990). Cuando un individuo hereda varios de estos genes mutados, su efecto acumulativo y el efecto del ambiente, contribuye a que esta persona sobrepase el umbral requerido. Posiblemente la enfermedad sea además genéticamente heterogénea: diferentes genes contribuyen al mismo fenotipo en diferentes familias y poblaciones (Dawson y Murray 1996).

\section{ESTUDIOS GENÉTICOS PARA LA LOCALIZACIÓN DE GENES DE SUSCEPTIBILIDAD}

La posible heterogeneidad genética, participación de varios genes de efecto menor y factores ambientales y la dificultad para hacer el diagnostico, pueden explicar la ausencia de resultados inequívocos en los estudios de ligamiento para las enfermedades mentales severas. (Mowry y Nancarrow 2001). Sin embargo, se han encontrado algunos loci con resultados significativos que posteriormente han sido replicados en otras poblaciones. Tales son los resultados en el cromosoma 1, 6, 8 y 13 (Farraone 2002). Es interesante que algunas de estas regiones también han sido asociadas al desorden bipolar (Cloninger 2002).

Algunas alternativas propuestas a los análisis clásicos de ligamiento en familias son realizar estudios no parametricos en parejas de hermanos afectados y estudios de asociación en poblaciones más homogéneas. En los estudios de parejas de hermanos afectados, se busca encontrar una región genómica que se herede en más del 50 por ciento esperado por azar. Los estudios de asociación buscan encontrar un alelo cuya frecuencia en los afectados sea significativamente diferente que entre los no afectados. En este caso por lo tanto, generalmente se utiliza una búsqueda dirigida a regiones o genes candidatos.

Al estudiar subgrupos más homogéneos de personas con la enfermedad se aumenta la probabilidad de que exista una alteración predominante en este subgrupo. Se ha mencionado el 
uso de poblaciones genéticamente aisladas, solo estudiar los casos severos de la enfermedad o encontrar fenotipos intermedios con un modo de herencia Mendeliano.

Por las anormalidades neuroquímicas y neuroanatómicas observadas en la enfermedad, una de las primeras teorías sobre la fisiopatología de la esquizofrenia propuso alteraciones en la neurotransmision.

\section{Sistema dopaminergico}

La dopamina es uno de los neurotransmisores que modula y regula el estado de ánimo y la cognición, particularmente la memoria, atención, sistemas endocrinos, funciones de ejecución, de recompensa y motoras (Exilien et al. 1999). La dopamina se inactiva al ser reabsorbida por la membrana presináptica o por medio de COMT. En la corteza prefrontal se regulan las funciones cognitivas que se encuentran alteradas en la esquizofrenia. Se ha sugerido que una deficiencia de la transmisión de dopamina en la corteza prefrontal puede ocasionar algunos de los síntomas de la enfermedad.

Los receptores dopaminergicos se pueden agrupar en dos familias D1 y D2. Dentro de la primera, se encuentran los receptores de dopamina D1 y D5. La segunda agrupa a los receptores D2, D3 y D4. La relación entre la eficacia del medicamento antipsicótico y la habilidad para bloquear los receptores de dopamina se encuentra restringida a la familia D2. Se sugiere que la esquizofrenia y el desorden bipolar están asociados con la hiperactividad de los receptores de la familia D2 (Wilson et al. 1998, Vallone et al. 2000).

El gen que codifica para catecol-o-metil transferasa (COMT) se encuentra en la región 22q11 (en la región del síndrome VCF, una deleción asociada a psicosis en el $50 \%$ de los casos). COMT es una enzima que metaboliza la dopamina liberada. Se han encontrado disminuciones significativas en los niveles de COMT en los eritrocitos de pacientes con esquizofrenia (McAllister y Summerall 2003, Norton et al. 2002). Estos resultados sugieren que una variación en la actividad de COMT puede tener efectos neurobiológicos específicos en la corteza prefrontal y por ende ser participe de la enfermedad (Egan et al. 2001). Sin embargo, no es posible excluir que el resultado sea efecto de los medicamentos.

\section{Sistema serotoninergico}

Se propone que una disfunción de los receptores $5-\mathrm{HT}_{2}$ puede estar asociada con la fisiopatología de la esquizofrenia debido a la gran afinidad de algunas drogas alucinógenas como el LSD por estos receptores. En investigaciones post-mortem de personas con esquizofrenia, se encuentra disminuida la densidad de los receptores 5- $\mathrm{HT}_{2}$ en la corteza prefrontal (Akhondzadeh 2001).

Se ha demostrado que las drogas o medicamentos que bloquean tanto los receptores de dopamina como los de serotonina son más eficaces en el tratamiento de la enfermedad. Los resultados de las drogas que modulan la actividad serotonérgica en el cerebro sugieren que al menos algunos de los síntomas de esquizofrenia son causados por un estado hiper-serotonérgico (Dean 2001).

\section{Sistema glutaminergico}

La teoría predominante actual es que la esquizofrenia es un desorden del neurodesarrollo lo que lleva a una conectividad anormal de las sinapsis. La disfunción de los receptores Nmetil-D-aspartato (NMDA) podría ser uno de los causantes de la enfermedad. G72, cuyo gen se localiza en el cromosoma 13q34, interactúa con la D-aminoácido oxidasa (DAAO) (situado en el cromosoma 12q24) para regular la señalización glutaminérgica por medio del receptor N-metil-D-aspartato (NMDA). Por medio de clonaje posicional y estudios de desequilibrio de ligamiento se mostró que los genes G72 y DAAO están asociados con un aumento en la susceptibilidad de la esquizofrenia. Algunas combinaciones de alelos G72 y DAAO aumentan significativamente el riesgo de sufrir esquizofrenia, más que la suma de sus efectos individuales, lo cual es evidencia de epistasis o 
interacciones gen-gen no aditivas (Harrison y Owen 2003, Chumakov et al. 2002).

Otro gen que se encuentra en la región de la deleción VCF y que podría estar involucrado con la esquizofrenia, es la prolina deshidrogenasa (PRODH2). Sirve como precursor del glutamato y además la prolina afecta potencialmente las sinapsis glutaminérgicas. Los ratones con el gen PRODH2 inactivo presentan anormalidades senso-motoras similares a las de esquizofrenia (Sawa y Zinder 2003).

\section{Otros genes candidatos}

1. NOTCH4: La proteína codificada por este gen es un receptor transmembrana que regula la diferenciación en todos los tipos de tejidos y organismos. Se localiza en el cromosoma $6 \mathrm{p} 21.3$. Las proteínas probablemente son cortadas antes de que se unan al ligando. Una o más de las formas intracelulares cortadas migran al núcleo donde regulan la transcripción génica junto a otras proteínas (Nye 2000). Las células que reciben una señal de notch4 asumen un destino y los que no la reciben asumen otro. Se conoce poco acerca de las funciones de NOTCH4 en humanos, pero los estudios en animales demuestran que la familia de proteínas NOTCH tienen la función de determinar el destino celular. Hibridaciones in situ en ratones mostraron que NOTCH4 se expresa en células endoteliales y su transcripción se detecta en el sistema nervioso en desarrollo. Algunos autores han encontrado asociación entre este gen y el riesgo de esquizofrenia; sin embargo, este resultado no ha sido replicado por otros (Wei y Hemmings 2000, Sklar et al. 2001, McGinnis et al. 2001).

2. Disbindina: La disbindina, localizada en el cromosoma $6 \mathrm{p} 22.3$, se expresa en las terminales presinápticas y puede participar en la formación y mantenimiento de las sinapsis y en la transducción de señales (Sawa y Zinder 2003). También puede disminuir la señalización de glutamato por medio de los receptores NMDA y se ha encontrado asociada al riesgo de la enfermedad. (Cloninger 2002, Straub et al. 2002).
3. Neuregulina 1: El gen de la neuregulina 1 se encuentra en el cromosoma 8p. Promueve la migración neuronal y la diferenciación celular. Se expresa en las vesículas sinápticas glutaminérgicas y actúa por medio de la vía de los receptores de NMDA en la expresión y activación del glutamato y otros receptores de neurotransmisores. Se han encontrado resultados positivos pos asociación y ligamiento para este gen (Stefansoon et al. 2003, McGuffin et al. 2003)

\section{ESTUDIOS EN COSTA RICA}

La genética de la esquizofrenia se estudia en Costa Rica desde 1997. Las estrategias han sido mediante (1) un estudio de ligamiento clásico con una familia multigeneracional y varios miembros afectados, (2) un estudio de parejas de hermanos afectados con la enfermedad, originarios de la región mesoamericana y (3) un estudio poblacional de asociación.

El diagnostico se realiza mediante el proceso de mejor estimado diagnostico: un equipo de expertos en esquizofrenia (dos por caso) estudian la información clínica recolectada para cada individuo para llegar a un diagnostico por consenso utilizando las pautas diagnosticas del DSMIV. La información clínica revisada consiste en: (1) una entrevista psiquiatrita semi-estructurada (DIGS) conducida por un psiquiatra entrenado en la entrevista y ciego al diagnostico, (2) la narrativa que el psiquiatra escribe luego de la entrevista, (3) una entrevista semi-estructurada (FIGS) sobre el paciente a un familiar cercano, conducida por un médico o psicólogo entrenado en la entrevista y (4) el resumen de los registros médicos del paciente incluyendo la consulta externa y las hospitalizaciones (Raventós et al. 1999, Medina et al. 2001).

Hemos encontrado que un $35 \%$ de los pacientes en el estudio no cumplen con las pautas diagnosticas del DSMIV de esquizofrenia. Estos pacientes han sido reclutados por tener un diagnostico de esquizofrenia en alguno de los hospitales nacionales lo cual sugiere que se 
sobre-diagnostica la esquizofrenia (Montero et al. 2002).

En cuanto al análisis genotípico, hemos encontrado resultados interesantes en los cromosomas 8, 13 y 18 (Balderas et al. 2001, Escamilla et al. 2001, Walss et al. 2002) lo cual confirma resultados encontrados en otras poblaciones. No concuerda con un tamizaje de todo el genoma conducido por otro grupo también en la población costarricense (DeLissi et al. 2002). Sin embargo, esto puede deberse a diferencias en los criterios de inclusión, las pautas diagnósticas o el análisis genealógicos entre los estudios.

Aunque por años los resultados positivos en estudios de mapeo para enfermedades mentales severas han sido seguidos por resultados negativos, estamos ante un momento alentador. Muchos de los resultados significativos han sido replicados en otras poblaciones y ya se cuenta con al menos 3 genes candidatos cuya evidencia es relativamente convincente.

\section{RESUMEN}

La esquizofrenia es una de las enfermedades mentales severas que afecta a un $1 \%$ de la población mundial. Es claro que en su etiología participan factores genéticos y ambientales. Sin embargo, la identificación de genes de susceptibilidad ha sido difícil y no hay resultados inequívocos. A pesar de esto, en los últimos años se han identificado un grupo de genes candidatos por su posible fisiopatología o por estudios de asociación y ligamiento. En este artículo se revisa el papel de estos genes y los resultados de los estudios realizados en Costa Rica por nuestro grupo.

\section{REFERENCIAS}

Adityanjee, YA. Aderibigbe, D. Theodoridis \& V.R. Vieweg. 1999. Dementia praecox to schizophrenia: The first 100 years. Psychiatry Clinical Neurosci. 53: 437-448.

Akhondzadeh, S. 2001. The 5-HT hypothesis of schizophrenia. Idrugs 4: 295-300.

American Psychiatric Association. 1994. Diagnostic and Statistical Manual of Mental Disorders. $4^{\text {th }}$ Edition. Washington DC.
Balderas, T.G., A.P. Montero, E. Benavides, S. Rodríguez, L. Almasy, H. Raventós \& MA. Escamilla. 2001. Linkage Disequilibrium Análisis of schizophrenia in the Costa Rican Population: Preliminary Findings on Chromosome 18. Am. J. Med. Genet. 105: 599

Bahn, S. 2002. Gene expression in bipolar disorder and schizophrenia: new approaches to old problems. Bipolar Disord. 4(Suppl. 1): 70-72.

Bassett, AS., EW. Chow, R. Weksberg \& L. Brzustowicz. 2002. Schizophrenia and Genetics: new insights. Curr. Psychiatry Rep. 4: 307-314.

Berrettini, W.H. 2000. Are schizophrenic and bipolar disorders related? A review of family and molecular studies. Biol. Psychiatry 48: 531-538.

Chumakov, I., M. Blumenfeld, O. Guerassimenko, L. Cavarec, M. Palicio, H. Abderrahim, L. Bougueleret, C. Barry, H. Tanaka, P. La Rosa, A. Puech, N. Tahri, A. Cohen-Akenine, S. Delabrosse, S. Lissarrague, F.P. Picard, K. Maurice, L. Essioux, et al. 2002. Genetic and physiological data implicating the new human gene G72 and the gene for D-amino acid oxidase in schizophrenia. Proc. Natl. Asoc. Sci. 99: 1367513680 .

Cloninger, C.R. 2002. The discovery of susceptibility genes for mental disorders Proc. Natl. Asoc. Sci. 99: 13365-13367.

Cowan, W.M., K.L. Kopnisky \& S.E. Imán. 2002. The Human Genome Proyect and its Impact on Psychiatry. Annu. Rev. Neurosci. 25:1-50.

Dawson, E. \& R. Murray. 1996. Schizophrenia: A gene at 6p?. Curr. Biol. 6: 268-271.

Dean, B. 2001. A predicted cortical serotonergic/cholinergic/gabaergic interface as a site of pathology in schizophrenia. Clin. Exp. Pharmacol. Physiol. 28: 74-78.

DeLisi L.E., A. Mesen, C. Rodriguez, A. Bertheau, B. LaPrade, M. Llach, S. Riondet, K. Razi, M. Relja, W. Byerley \& R. Sherrington. 2002. Genome-wide scan for linkage to schizophrenia in a Spanish-origin cohort from Costa Rica. Am. J. Med. Genet. 114: 497-508.

Egan, M.F., T.E. Goldberg, B.S. Kolachana, J.H. Callicott, C.M. Mazzanti, R.E. Straub, D. Goldman \& DR. Weinberg. 2001. Effect of COMT Val 108/158 Met genotype on frontal lobe function and risk for schizophrenia. Proc. Natl. Asoc. Sci. 98: 6917-6922.

Emilien, G., J.M. Maloteaux, M Geurts, K. Hoogenberg \& S. Cragg. 1999. Dopamine receptors- Physiological understanding to therapeutic intervention potential. Pharmacol. Ther. 84: 133-156. 
Escamilla, M.A., H. Raventos, L. Almasy, P. Montero, T. Balderas, S. Rodríguez \& D. Levinson. 2001. Linkage Disequilibrium Analisis of Schizophrenia and Schizoaffective Disorder in the Costa Rican Population: Preliminary findings on chromosome 13. Am. J. Med. Genet. 105: 599.

Evans, K.L., W.J. Muir, D. Blackwood \& D.J. Porteous. 2001. Nuts and bolts of psychiatric genetics: building on the Human Genome Proyect. Trends Genet. 17: 35-40.

Faraone, SV., L. Taylor \& MT. Tsuang. 2002. The molecular genetics of schizophrenia: an emerging consensus. Expert Rev. Mol. Med. 2002: 1-3.

Harrison, P.J. \& M.J. Owen. 2003. Genes for schizophrenia? Recent findings and their pathophysiological implications. Lancet 361: 417-419.

Kelly, J. \& RM. Murria. 2000. What risk factors tell us about the causes of schizophrenia and related psychoses. Curr. Psychiatry Rep. 2: 378-385.

Liu, H., G.R. Abecasis, S.C. Heath, A. Knowles, S. Demars, Y.J. Chen, J.L. Roos, J.L. Rapoport, J.A. Gogos \& M. Karayiorgou. 2002. Genetic Variation in the 22q11 locus and the susceptibility to schizophrenia. Proc. Natl. Asoc. Sci. 99: 16859-16864.

Lui, H., SC. Heath, C. Sobin, J.L. Roos, B.L. Galke, M.L. Blundell, M. Lenane, B. Robertson, EM. Wijsman, JL. Rapoport, JA. Gogos \& M. Karayiurgou. 2002. Genetic Variation at the 22q11 PRODH2/DGCR6 locus presents an unusual pattern and increases susceptibility to schizophrenia. Proc. Natl. Asoc. Sci. 99: 3717-3722.

Malaspina, D., S. Harlap, S. Fennig, D. Heiman, D. Nahon, D. Feldman \& E.S. Susser. 2001. Advancing Paternal Age and the Risk of Schizophrenia. Arch. Gen. Psychiatry. 58: 361-367.

McAllister, T.W. \& L. Summerall. 2003. Genetic Polymorphisms in the Expression and Treatment of Neuropsychiatric Disorders. Curr. Psychiatry Rep. 5: 400-409.

McGinnis, R.E., H. Fox, P. Yates, L.A. Cameron, M.R. Barnes, I.C. Gray, N.K. Spurr, O. Hurko \& D. St.Clair. 2001. Failure to confirm NOTCH4 association with schizophrenia in a large population-based sample from Scotland. Nat. Genet. 28: 128-129.

McGuffin, P., K. Tandom \& A. Corsico. 2003. Linkage and Association Studies of Schizophrenia. Curr. Psychiatry Rep. 5: 121-127.

Montero, A.P., T. Balderas, M. Pereira, A. Lizano, R. Armas, R. Medina, S. Contreras, A. Dassori, I. Katz, D.
Levinson, J.J. Garbarz, M. Escamilla \& H. Raventós. 2002. Resultados de la categorización de una muestra de pacientes con diagnóstico de egreso de esquizofrenia. Acta Méd. Cost. 44(2): 74-78.

Mowry, BJ. \& DJ. Nancarrow. 2001. Molecular Genetics of Schizophrenia. Clin. Exp. Pharmacol. Physiol. 28: 66-69.

Medina, R.A., M.A. Escamilla, R. Armas, D. Levinson, A. Dassori, S. Contreras \& H. Raventos. 2001. Diagnostic Reliability in a study of Schizophrenic subjects from the Costa Rican Population. Am. J. Med. Genet. 105(7): 599.

Norton, N., G. Kirov, S. Zammit, G. Jones, S. Jones, R. Owen, M. Krawczak, NM. Williams, MC. O’Donovan \& M.J. Owen. 2002. Schizophrenia and Functional Polymorphisms in the MAOA and COMT Genes: No Evidence for Association or Epistasis. Am. J. Med. Genet. 114: 491-496.

Nye, J.S. 2000. The Notch Proteins. Curr. Biol. 9(4): R118.

Raventós, H., M. Escamilla, P. Montero, R. Armas, I. Katz, D. Levinson, C. Mathews, S. Vinogradov, J.J. Garbarz, A. Hernandez, R. Castro \& M. Quesada. 1999. Phenotypic Definition of Schizophrenia in the Costa Rican Population: Diagnostic and Assessment Issues. Mol. Psychiatry 4 (Supp. 1): S41.

Risch, N. 1990. Linkage strategies for genetically complex traits. I. Multilocus models. Am. J. Hum. Genet. 46(2): 222-228.

Sawa, A. \& S.H. Snyder. 2003. Schizophrenia: neural mechanisms for novel therapies. Mol. Med. 9(1-2): 3-9.

Sklar, P., S.G. Schwab, N.M. Williams, M. Daly, S. Schaffner, W. Maier, M. Albus, M. Trixler, P. Eichhammer, B. Lerer, J. Hallmayer, N. Norton, H. Williams, S. Zammit, AG. Cardno, S. Jones, G. McCarthy, V. Milanova, G. Kirov, MC. O’Donovan, ES. Lander, MJ. Owen \& DB. Wildenauer. 2001 Association analysis of NOTCH4 loci in schizophrenia using family and population-based controls. Nat. Genet. 28: 126-128.

Stefansson, H., J. Sarginson, A. Kong, P. Yates, V. Steinthorsdottir, E. Gudfinnsson, S. Gunnarsdottir, N. Walker, H. Petursson, C. Crombie, A. Ingason, JR. Gulcher, K.

Stefansson \& D. St.Clair. 2003. Association od Neuregulin 1 with Schizophrenia Confirmed in a Scottish Population. Am. J. Hum. Genet. 72: 83-87.

Straub, RE., Y. Jiang, C.J. MacLean, Y. Ma, B.T. Webb, M.V. Myakishev, C. Harris-Kerr, B. Wormley, H. 
Sadek, B. Kadambi, A.J. Cesare, A. Gibberman, X. Wang, F.A. O'Neill, D. Walsh \& K.S. Kendler. 2002. Genetic Variation in the 6 p22.3 Gene DTNBP1, the Human Ortholog of the Mouse Dysbindin Gene, is Associated with Schizophrenia. Am. J. Hum. Genet. 71: $337-348$.

Torrey, E.F., A.E. Bowler, E.H. Taylor \& II. Gottesman. 1994. Schizophrenia and Manic-Depressive Disorder: The Biological Roots of Mental Illness as Revealed by the Landmark Study of Identical Twins. Basic Books. New York, NY. 274 p.

Vallone, D., R. Picetti \& E. Borrelli. 2000. Structure and function of dopamine receptors. Neurosci. Biobehav. Rev. 24: 125-132.
Walss-Bass, C., M.A. Escamilla, H. Raventos, A.P. Montero, R. Armas, A. Dassori, S. Contreras, D. Levinson, E. Benavides, S. Rodriguez, R. Pereira, T.G. Balderas, M. Pereira, I. Atmella, R. Medina, L. Gutierrez, R. Castro, M. Hernandez, J.J. Garbarz, I. Katz \& L. Almasy. 2002. Genome wide linkage disequilibrium analysis of schizophrenia in the Costa Rican population: preliminary findings on chromosomes 1, 8, 13 and 22. Am. J. Med. Genet. 114(7): 877.

Wei, J. \& G.P. Hemmings. 2000. The NOTCH4 locus is associated with susceptibility to schizofrenia. Nat. Genet. 25: 376-377.

Wilson, J.M., S. Sanyal \& H. Vantol. 1998. Dopamine D2 and D4 receptor ligands: relation to antipsichotic action. Eur. J. Pharmacol. 351(3): 273-286. 